\title{
Spin Nernst effect and intrinsic magnetization in two-dimensional Dirac materials
}

\author{
V.P. Gusynin and S.G. Sharapov \\ Bogolyubov Institute for Theoretical Physics, National Academy of Sciences of Ukraine \\ 14-b Metrologicheskaya Str., Kiev 03680, Ukraine \\ E-mail:vgusynin@bitp.kiev.ua \\ A.A. Varlamov \\ CNR-SPIN, University “Tor Vergata”, Viale del Politecnico 1, Rome I-00133, Italy
}

Received November 12, 2014, published online March 23, 2015

\begin{abstract}
We begin with a brief description of the role of the Nernst-Ettingshausen effect in the studies of the hightemperature superconductors and Dirac materials such as graphene. The theoretical analysis of the NE effect is involved because the standard Kubo formalism has to be modified by the presence of magnetization currents in order to satisfy the third law of thermodynamics. A new generation of the low-buckled Dirac materials is expected to have a strong spin Nernst effect that represents the spintronics analog of the NE effect. These Dirac materials can be considered as made of two independent electron subsystems of the two-component gapped Dirac fermions. For each subsystem the gap breaks a time-reversal symmetry and thus plays a role of an effective magnetic field. We explicitly demonstrate how the correct thermoelectric coefficient emerges both by the explicit calculation of the magnetization and by a formal cancelation in the modified Kubo formula. We conclude by showing that the nontrivial dependences of the spin Nersnt signal on the carrier concentration and electric field applied are expected in silicene and other low-buckled Dirac materials.
\end{abstract}

PACS: 72.25.Dc Spin polarized transport in semiconductors;

65.80.Ck Thermal properties of graphene;

72.80.Vp Electronic transport in graphene;

81.05.ue Graphene.

Keywords: Dirac materials, spin transport, Nernst effect, magnetization.

\section{Introduction}

This work is devoted to 70th anniversary of our colleague, teacher, and a remarkable physicist Vadym Mykhailovych Loktev. The field of his scientific interests is extremely wide and spreads from molecular and magnetic excitons to the mechanisms of high-temperature superconductivity (HTSC). Bearing in mind such a reader of our contribution, we begin with presenting an overview of the studies of the Nernst-Ettingshausen (NE) effect. The main text is based on our paper [1].

The thermoelectric and thermomagnetic phenomena discovered in the nineteen century turned out unexpectedly to be in the spotlight at the beginning of the twenty first one. First of all, from the practical point of view, control of heat fluxes and minimization of the related losses are important factors in designing of modern elements of nanoelecronics. For instance, the experiments [2] indicate that thermoelectric effect in graphene accounts for up to $30 \%$ of the contact temperature changes and thus it can play significant role in cooling down of nanostructures based on such systems.

During the last decade, the Nernst-Ettingshausen effect also attracts an enhanced attention of the superconductive research community. First indications of a sizeable NE effect in a wide range of temperatures above the critical temperature was given by Palstra et al. [3] (see also [4,5]) who detected it in the optimally doped YBCO samples at temperatures up to $10 \mathrm{~K}$ above the phase transition. The discovery of a giant NE effect (hundred times larger than its value in conventional metals) in the pseudogap state of $\mathrm{La}_{2-} \mathrm{Sr}_{x} \mathrm{CuO}_{4}$ [6] was a next milestone followed by the similar finding (with a $10^{3}$ enhancement in magnitude in the wide range of temperatures) in the low-temperature super- 
conductor $\mathrm{Nb}_{0.15} \mathrm{Si}_{0.85}$ [7]. These observations have been especially surprising in view of the previously collected data on the magnitude of the Seebeck coefficient $S_{f l}$ in the fluctuative regime of a superconductor, undergoing close to $T_{C}$ a weak singular decrease but remaining of the same order as in the normal phase above $T_{C}$ [8-10].

The theoretical description of fluctuation contributions to the thermoelectric coefficients remains complex and controversial. In what concerns the fluctuation contribution to the NE coefficient $v_{f l}$, it was initially found in the framework of the GL approach [11]. In contrast to its value in the normal phase, $v_{f l}$ does not contain the electron-hole asymmetry smallness and was found to be much more singular than $S_{f l}: v_{f l} \sim T_{\mathrm{c}} /\left(T-T_{\mathrm{C}}\right)$. After the experimental findings of Ref. 6 the problem was revisited in Ref. 12, where the linear response theory result of Ref. 11 was reproduced and additionally the importance of magnetization currents was emphasized. Indeed, half a century ago Obraztsov noticed [13] that the Onsager principle applied to the thermoelectric tensor in the presence of magnetic fields can be satisfied only if accounting for the nonhomogeneous magnetization currents affected by the temperature gradient. These currents contribute to the NE coefficient as well. However, in normal metals their contribution appears to be negligible and it does not change the classical Sondheimer result for the NE coefficient [14], obtained using the transport equation approach. Nevertheless, the role of magnetization currents becomes crucial in the regime of quantum fluctuations: accounting for only the $\mathrm{Ku}-$ bo-like response contribution to the heat flow [15] results in the violation of the third law of thermodynamics and only taking the fluctuative Meissner magnetization above $H_{c 2}(0)$ into consideration regularizes the situation [16]. Similar contradictions to the thermodynamics laws were found also in studies of thermomagnetic effects in other, very different from superconductor, systems [1,17] and in each case the magnetization currents contribution helped resolving the paradoxes.

This revival of interest to the thermoelectric and thermomagnetic effects also in full extend concerns the studies of new Dirac type materials. For example, a huge compared to the nonmagnetic metals Seebeck and Nernst effects $\sim 50-100 \mu \mathrm{V} / \mathrm{K}$ at room temperature were observed [18-21] in graphene, where the Dirac point can be crossed by tuning the position of the chemical potential $\mu$, Moreover, Seebeck and NE effects can be further enhanced and controlled by opening a gap in the quasiparticle spectrum [22-24].

In this context, the synthesis of silicene [25-32], a monolayer of silicon atoms forming a two-dimensional low-buckled lattice, boosted theoretical studies of a wide class of new Dirac materials. The honeycomb lattice of silicene can be described as in graphene in terms of two triangular sublattices. However, a larger ionic size of silicon atoms results in buckling of the 2D lattice. Accordingly, the sites on the two sublattices are vertically separated at a distance $2 d \approx 0.46 \AA$. Consequently, silicene is expected [33-36] to have a strong intrinsic spin-orbit interaction that results in a sizable spin-orbit gap, $\Delta_{S O}$, in the quasiparticle spectrum opened at the Dirac points. Moreover, by applying the electric field $E_{z}$ perpendicular to the plane, it is possible to create the on-site potential difference between the two sublattices opening the additional gap, $\Delta_{Z}=E_{Z} d$, in the quasiparticle spectrum.

Similar structure and properties are also expected in $2 \mathrm{D}$ sheets of Ge, Sn, P and Pb atoms $[37,38]$ (the first three materials are coined as germanene, stanene and phosphorene).

Due to nonzero spin-orbit gap $\Delta_{S O}$ the quantum spin Hall (QSH) effect [39] becomes experimentally accessible in silicene. The latter is fundamentally related to the anomalous Hall effect in ferromagnets [40]. The analogy between thermomagnetic phenomena in graphene and spintronics of the low-buckled Dirac materials shows that the latter should also be very promising for the investigation of the spin caloritronics phenomena [41]. Among these, there is a particular interest in the off-diagonal spin Nernst (SN) effect, an analogue of the NE effect in a normal conductor subjected to a magnetic field. It is the spinorbit gap $\Delta_{S O}$ that plays the role of an effective magnetic field that generates the SN effect even in the absence of a real magnetic field in the new Dirac materials. In this paper, we will show that due to the large value of $\Delta_{S O} \sim 10 \mathrm{meV}$ the off-diagonal thermospin coefficient, $\beta_{x y}^{S_{Z}}$, is indeed expected to be huge in these materials while at the same time being nontrivially dependent on the chemical potential $\mu$ and electric field $E_{z}$.

The paper is organized as follows. We begin by presenting in Sec. 2 the model describing silicene and the basic model of the gapped two-component Dirac fermions. The relationship between thermospin transport coefficients for silicene and thermoelectric coefficients for the basic model is considered. The qualitative analysis of the thermoelectric coefficient $\beta_{x y}$ is given using the Mott relation in Sec. 3 . The specifics of the off-diagonal thermal transport and the necessity to consider magnetization currents are discussed in Sec. 4. The magnetization of the gapped Dirac fermions is considered in Sec. 5. In particular, in Sec. 5.1 we present an explicit expression for magnetization and in Sec. 5.2 show its role in the restoration of the third law of thermodynamics. The final results for two-component Dirac fermions and silicene are presented in Sec. 6. The main results are summarized in Sec. 7, where also the possibility of the experimental observation of the SN in silicene is discussed. The full electric current vertex is found in Appendix A.

\section{Models}

\subsection{Model of silicene}

The low-energy physics of silicene is described by the Hamiltonian density [34-36] 


$$
\begin{gathered}
\mathcal{H}_{\xi}=\sigma_{0} \otimes\left[\hbar v_{F}\left(\xi k_{x} \tau_{1}+k_{y} \tau_{2}\right)+\Delta_{z} \tau_{3}-\mu \tau_{0}\right]- \\
-\xi \Delta_{S O} \sigma_{3} \otimes \tau_{3},
\end{gathered}
$$

where the Pauli matrices $\boldsymbol{\tau}$ and $\boldsymbol{\sigma}$, and the unit matrices, $\tau_{0}$ and $\sigma_{0}$ act in the sublattice and spin spaces, respectively, and the wavevector $\mathbf{k}$ is measured from the $\mathbf{K}_{\xi}$ points (valleys) with $\xi= \pm$. Here we neglected the small Rashba interaction [42]. The Hamiltonian (1), describes four kinds (two identical pairs) of the noninteracting massive (gapped) Dirac quasiparticles with the masses $\Delta_{\xi \sigma} / v_{F}^{2}$, where $v_{F}$ is the Fermi velocity, $\sigma(= \pm)$ is the spin, and the valley-dependent gap $\Delta_{\xi \sigma}=\Delta_{Z}-\xi \sigma \Delta_{S O}$.

The QSH effect in silicene occurs due to the presence of two subsystems with $\sigma= \pm$ each exhibiting the quantum Hall effect. The corresponding chiral edge states are spin-polarized and form a time-reversed pair to recover the overall time-reversal symmetry (the Kane-Mele scenario [39]). The spin Hall conductivity can be expressed $[43,44]$ in terms of the electric Hall conductivity $\sigma_{x y}(\Delta)$ for the two-component Dirac fermions with the gap $\Delta$ (see the Hamiltonian (3) below) by the relation

$$
\sigma_{x y}^{S_{Z}}=-\frac{\hbar}{2 e} \sum_{\xi, \sigma= \pm} \xi \sigma \sigma_{x y}\left(\Delta \rightarrow \Delta_{\xi \sigma}\right) .
$$

The factor $-\hbar / 2 e$ indicates that in the off-diagonal correlation function of the two electric currents, one electric current is replaced by the spin current.

Being subjected to the temperature gradient $\nabla T$, the spin-polarized chiral edge states loose their time-reversal symmetry and the spin current $\mathbf{j}^{s}$ flows. The latter is related to $\nabla T$ by means of the thermospin tensor, $\hat{\beta}^{s}$, via [41] $\mathbf{j}^{s}=-\hat{\beta}^{s} \nabla T$. Analogously to the spin Hall conductivity, the off-diagonal component $\beta_{x y}^{S_{Z}}$ can be obtained from (2) by the substitution $\sigma_{x y}^{S_{z}} \rightarrow \beta_{x y}^{S_{z}}$ and $\sigma_{x y}(\Delta) \rightarrow \beta_{x y}(\Delta)$, where $\beta_{x y}(\Delta)$ is the standard thermoelectric coefficient for the two-component Dirac fermions. Thus in the absence of valley mixing, the study of the spin transport coefficient is reduced to an investigation of the electric transport for the two-component gapped Dirac fermions.

\subsection{Model of two-component Dirac fermions}

The corresponding Hamiltonian density is

$$
\mathcal{H}=\hbar v_{F}\left(k_{x} \tau_{1}+k_{y} \tau_{2}\right)+\Delta \tau_{3}-\mu \tau_{0}
$$

This model with broken time-reversal symmetry provides a simple realization of the anomalous Hall and thermoelectric effects. Its main merit is the possibility of obtaining simple approximate analytical expressions in the presence of spin-independent random potential with Gaussian correlations [40,43,45]. The two-component fermion model (3) is considered in Secs. 3, 4 and 5.

\section{Qualitative analysis}

A qualitative evaluation of the thermoelectric coefficient $\beta_{x y}(\Delta)$ can be obtained basing on the Mott relation,

$$
\beta_{x y}=-\frac{\pi^{2} k_{B}^{2}}{3 e} T \frac{\partial \sigma_{x y}(\mu, \Delta, T=0)}{\partial \mu},
$$

where $k_{B}$ is the Boltzmann constant. In the clean limit at $T=0$ one finds $[43,45]$

$$
\sigma_{x y}=-\frac{e^{2} \operatorname{sgn}(\Delta)}{4 \pi \hbar}\left\{\begin{array}{cc}
1, & |\mu| \leq|\Delta|, \\
|\Delta| /|\mu|, & |\mu|>|\Delta|,
\end{array}\right.
$$

from which we can draw two conclusions: i) For $|\mu|>|\Delta|$, we obtain that $\beta_{x y}=-\left(k_{B} / e\right)\left(\pi e^{2} / 12 \hbar\right)\left(\Delta \operatorname{sgn}(\mu) / \mu^{2}\right) k_{B} T$. Then the Nernst signal $e_{y}(T) \equiv-E_{y} / \nabla_{x} T$, where $E_{y}$ is the electric field in $y$-direction, can be estimated as

$$
e_{y}(T) \approx \frac{\beta_{x y}}{\sigma_{x x}}=-\left(\frac{k_{B}}{e}\right) \frac{\pi e^{2}}{12 \hbar \sigma_{x x}} \frac{k_{B} T \Delta \operatorname{sgn}(\mu)}{\mu^{2}} .
$$

Here we assumed that the diagonal conductivity is much larger than the Hall conductivity, $\sigma_{x x}>\left|\sigma_{x y}\right|$.

The main feature of the Dirac materials is that the value of the chemical potential $\mu$ can be tuned as close as possible to the regime with $e_{y}(T) \sim k_{B} / e \sim 86 \mu \mathrm{V} / \mathrm{K}$. This is exactly how one gains from 3 to 4 orders of magnitude in the Nernst signal as compared to the normal nonmagnetic metals, where $e_{y}$ is negligibly small ( $10 \mathrm{nV} / \mathrm{K}$ per Tesla).

ii) Our simple estimate also shows that Mott's formula fails near $|\mu|=|\Delta|$ when the conductivity $\sigma_{x y}(\mu, \Delta, T=0)$ changes abruptly. Indeed, as discussed recently in [23] (see also references therein), when the gap is present in the quasiparticle spectrum, one should use the microscopic approach. The same is true for the SN effect: as of yet it has been studied mostly using a formula analogous to Mott's formula written for the spin conductivity [46-48] (see also the numerical study [49] based on the LandauerButtiker formula).

\section{Modified Kubo formula}

The study of the off-diagonal thermal transport in the framework of the Kubo formalism is a delicate issue. It was firstly understood 50 years ago by Obraztsov [50] that, in conjunction with the Kubo-like response on the temperature gradient, magnetization currents must be taken into account in order to satisfy the Onsager principle of the symmetry of the kinetic coefficients. It is worthwhile to mention that this problem has been readdressed in almost every decade [51-55] due to its importance for the quantum Hall effect, NE in fluctuating superconductors, etc. In the problem under consideration the account for magnetization currents turns out to be crucial not only in order to get the correct coefficient in $\beta_{x y}$ for the two-component 
gapped Dirac fermions, but first and foremost for the validity of the third law of thermodynamics.

We will show below, that the mere calculation of

$$
\tilde{\beta}_{x y}=-\frac{\hbar}{T} \lim _{\omega \rightarrow 0} \frac{Q_{x y}^{e q(R)}(\omega)}{\omega}
$$

(here $Q_{x y}^{e q(R)}$ is the retarded response function of electric and heat currents) in the Kubo formalism results in the expression that in the low-temperature limit is presented in the form of the Laurent series: $a_{-1} / T+a_{0}+a_{1} T+\cdots$. At the same time, it is clear that at zero temperature the thermoelectric tensor must become zero: it describes the transport of entropy, which, in accordance with the third law of thermodynamics, becomes zero when $T \rightarrow 0$. In the presence of an effective magnetic field, the off-diagonal thermal transport coefficient $\tilde{\beta}_{x y}$ has to be enriched [50] by including the magnetization $M_{z}$ term, so that

$$
\beta_{x y}=\tilde{\beta}_{x y}+\frac{c M_{z}}{T} .
$$

Here $c$ is the velocity of light. The latter exactly cancels out both $a_{-1} / T$ and $a_{0}$ terms in the complete expression for $\beta_{x y}$, making it proportional to the absolute temperature in the vicinity of $T=0$ and reconciling the theory with the basic principles of thermodynamics.

The above-mentioned electric-heat currents linear response function in the Matsubara representation can be presented as the bubble containing two full Green's functions (GF), one full and one bare vertices [56] (see Fig. 1)

$$
\begin{aligned}
& Q_{\alpha \beta}^{e q}\left(\Omega_{m}\right)=\sum_{\epsilon_{n}} \int \frac{d^{2} k}{(2 \pi)^{2}} \operatorname{tr}\left[\Upsilon_{\alpha}^{(e)}\left(\epsilon_{n}+\Omega_{m}, \epsilon_{n}\right) \times\right. \\
& \left.\times G\left(\epsilon_{n}+\Omega_{m}, \mathbf{k}\right) \Upsilon_{\beta}^{(q)}\left(\epsilon_{n}, \epsilon_{n}+\Omega_{m}\right) G\left(\epsilon_{n}, \mathbf{k}\right)\right] .
\end{aligned}
$$

Here

$$
G\left(\epsilon_{n}, \mathbf{k}\right)=\frac{1}{i \epsilon_{n} \tau_{0}-\mathcal{H}-\Sigma\left(i \epsilon_{n}\right)}
$$

is the two-component gapped Dirac fermions' GF with the self-energy

$$
\Sigma^{R}(\epsilon)=-i \Gamma_{0}(\epsilon) \tau_{0}-i \Gamma_{1}(\epsilon) \tau_{3},
$$

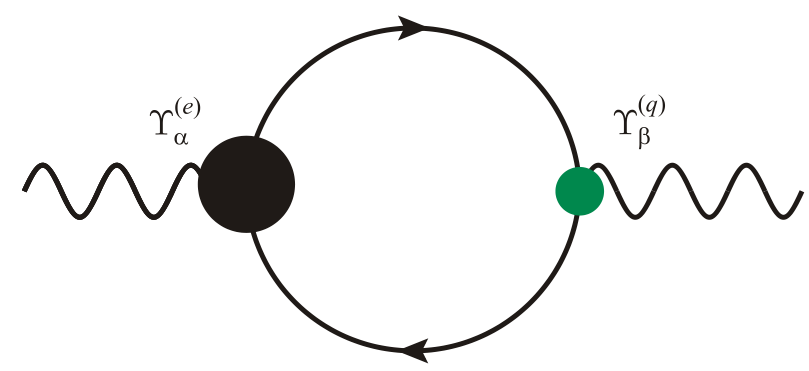

Fig. 1. (Color online) The thermoelectric tensor in terms of Feynman diagram.

$$
\Upsilon_{\beta}^{(q)}\left(\epsilon_{n}, \epsilon_{n}+\Omega_{m}\right)=i\left(\epsilon_{n}+\Omega_{m} / 2\right) v_{F} \tau_{\beta}
$$

is the heat current vertex for non-interacting fermions, and finally, $\Upsilon_{\alpha}^{(e)}\left(\epsilon_{n}+\Omega_{k}, \epsilon_{n}\right)$ is the electric current vertex renormalized by the impurity scattering. $\Gamma_{0}(\epsilon)$ and $\Gamma_{1}(\epsilon)$ are the corresponding scattering rates. These scattering rates along with the full electric current vertex are found in Appendix A.

In order to see the role of magnetization it is very instructive to start our analysis from the clean limit, $\Sigma^{R}(\epsilon)=-i \Gamma_{0}(\epsilon) \tau_{0}$ with $\Gamma_{0}(\varepsilon)=\Gamma_{0} \rightarrow 0$ and the bare vertex $\Upsilon_{\alpha}^{(e)}\left(\epsilon_{n}+\Omega_{m}, \epsilon_{n}\right)=-e v_{F} \tau_{\alpha}$. In this case one can explicitly calculate the Kubo and magnetization parts of the off-diagonal thermoelectric coefficient $\beta_{x y}$. We obtain that the Kubo part (7) can be written in the form

$$
\tilde{\beta}_{\alpha \beta}=\tilde{\beta}_{\alpha \beta}^{(1)}+\tilde{\beta}_{\alpha \beta}^{(2)}
$$

with

$$
\begin{gathered}
\tilde{\beta}_{\alpha \beta}^{(1)}=\frac{e \hbar v_{F}^{2}}{4 \pi T} \int_{-\infty}^{\infty} d \epsilon\left(-\frac{\partial f(\epsilon)}{\partial \epsilon}\right) \epsilon \times \\
\times \operatorname{Tr}\left[\tau_{\alpha} G^{R} \tau_{\beta}\left(G^{R}-G^{A}\right)-\tau_{\alpha}\left(G^{R}-G^{A}\right) \tau_{\beta} G^{A}\right]
\end{gathered}
$$

and

$$
\begin{gathered}
\tilde{\beta}_{\alpha \beta}^{(2)}=\frac{e \hbar v_{F}^{2}}{4 \pi T} \int_{-\infty}^{\infty} d \epsilon f(\epsilon) \epsilon \operatorname{Tr}\left[\tau_{\alpha} \frac{d G^{R}}{d \varepsilon} \tau_{\beta} G^{R}-\right. \\
\left.-\tau_{\alpha} G^{R} \tau_{\beta} \frac{d G^{R}}{d \varepsilon}-\tau_{\alpha} \frac{d G^{A}}{d \varepsilon} \tau_{\beta} G^{A}+\tau_{\alpha} G^{A} \tau_{\beta} \frac{d G^{A}}{d \varepsilon}\right],
\end{gathered}
$$

where the retarded (advanced) GF is $G^{R, A}(\epsilon, \mathbf{k})=$ $=G\left(\epsilon_{n} \rightarrow \epsilon \pm i 0, \mathbf{k}\right)$ and $\operatorname{Tr}$ denotes the integration over $\mathbf{k}$ and, as in (9), the trace over sublattice indices. The Kubo contribution (11) along with the standard term, $\tilde{\beta}_{\alpha \beta}^{(1)}$ containing the derivative of the Fermi distribution, $\partial f(\epsilon) / \partial \epsilon$, also contains the term, $\tilde{\beta}_{\alpha \beta}^{(2)}$, with the integral containing the Fermi distribution, $f(\varepsilon)=1 /\left[\exp \left(\varepsilon / k_{B} T\right)+1\right]$ itself. It is the latter that in the low-temperatures limit $T \rightarrow 0$ produces the diverging part,

$$
\tilde{\beta}_{x y}=-\frac{e}{4 \pi \hbar T}[\Delta \operatorname{sgn}(\mu) \theta(|\mu|-|\Delta|)+\mu \operatorname{sgn}(\Delta) \theta(|\Delta|-|\mu|)] \text {. }
$$

Thus we see that even in the absence of a real external magnetic field $B$, the calculation of $\beta_{x y}$ in the model (3) using the Kubo formula reveals a difficulty very similar to the problem solved by Obraztsov [50]. It is the gap $\Delta$ that plays the role of the external magnetic field in Eq. (14). Below we will explicitly show that accounting for the magnetization term $c M_{z} / T$ removes the divergence in $\beta_{x y}$. 


\section{Magnetization as the response on the gap and validity of the third law of thermodynamics}

Since the Hamiltonian (3) breaks the time-reversal symmetry, the intrinsic magnetization (magnetic moment per unit volume) [51-53]

$$
M_{z}=\frac{e v_{F}}{2 c} \int_{-\infty}^{\infty} d \epsilon f(\epsilon) \operatorname{Tr}\left[\delta\left(\epsilon \tau_{0}-\mathcal{H}\right)\left(\hat{r}_{\beta} \tau_{\alpha}-\hat{r}_{\alpha} \tau_{\beta}\right)\right]
$$

is indeed expected to be nonzero. However, an attempt to calculate $M_{z}$ from the definition (15) fails due to the difficulties that were resolved only recently (see Ref. 59 and references therein). It is the unboundedness of the coordinate operator $\hat{r}_{\alpha}$ with $\alpha=x, y$ that does not allow one to derive $M_{z}$ directly.

\subsection{Explicit expression for magnetization and its relation to the Berry phase}

To overcome this problem, we start from the GF of the charge carriers in the magnetic field written in the coordinate representation. Having the GF, it is already straightforward to calculate the carrier density $\rho(\mu, T, B)$. The thermodynamic potential $\Omega(\mu, T, B)$ can be obtained by integrating the relationship $\rho=-\partial \Omega / \partial \mu$ over $\mu$.

Finally, the magnetization is derived as $M_{z}=-\partial \Omega / \partial B$ (all the details are provided in Appendix of Ref. 1) and we obtain

$$
\begin{gathered}
M_{Z}=\frac{e T \operatorname{sgn}(\Delta)}{2 \pi \hbar c} \operatorname{Re}\left[\ln \Gamma\left(\frac{1}{2}+\frac{\Gamma_{0}-i(\mu-|\Delta|)}{2 \pi T}\right)-\right. \\
-\ln \Gamma\left(\frac{1}{2}+\frac{\Gamma_{0}-i(\mu+|\Delta|)}{2 \pi T}\right)-\ln \Gamma\left(\frac{1}{2}+\frac{\Gamma_{0}+i|\Delta|}{2 \pi T}\right)+ \\
\left.+\ln \Gamma\left(\frac{1}{2}+\frac{\Gamma_{0}-i|\Delta|}{2 \pi T}\right)\right]
\end{gathered}
$$

where $\Gamma(z)$ is the gamma function. Let us stress that since the time-reversal symmetry is broken, a finite fieldindependent contribution to $M_{z}$ appears.

It follows from Eq. (16) that for $T>>\Gamma_{0}$, the magnetization takes the especially simple form

$$
\frac{M_{z}}{T}=\frac{\operatorname{esgn}(\Delta)}{4 \pi \hbar c}\left[\ln \cosh \frac{\mu+|\Delta|}{2 k_{B} T}-\ln \cosh \frac{\mu-|\Delta|}{2 k_{B} T}\right] .
$$

Remarkably, in the limit $T \rightarrow 0$ the asymptotic expression of Eq. (17) reduces to Eq. (14) but with the opposite sign. This restores the validity of the third law of thermodynamics. Since $\beta_{x y}$ describes the transversal entropy transport it must identically become zero at $T=0$. Equation (17) illustrates in a spectacular way how the gap $\Delta$ induces the anomalous magnetic moment.

Note also that Eq. (17) for the orbital magnetization can be rewritten in the form of the general expression for the magnetization that was suggested in the studies of the role of the Berry phase in the anomalous thermoelectric transport (see, for example, Eqs. (6) and (14) in [55,60], respectively, and Refs. 61, 62)

$$
\begin{aligned}
\mathbf{M} & =\sum_{n} \int \frac{d \mathbf{k}}{(2 \pi)^{2}}\left[\mathbf{m}_{n}(\mathbf{k}) f\left(\epsilon_{n}(\mathbf{k})-\mu\right)+\right. \\
& \left.+\frac{e}{\hbar} \mathbf{\Omega}_{n}(\mathbf{k}) \frac{1}{\beta} \ln \left(1+\mathrm{e}^{-\beta\left(\epsilon_{n}(\mathbf{k})-\mu\right)}\right)\right] .
\end{aligned}
$$

Here

$$
\mathbf{m}_{n}(\mathbf{k})=-\frac{i e}{2 \hbar c}\left\langle\nabla_{k} u_{n k}\left|\times\left[\mathcal{H}(\mathbf{k})-\epsilon_{n}(\mathbf{k})\right]\right| \nabla_{k} u_{n k}\right\rangle
$$

is the orbital magnetic moment of the state $(n, \mathbf{k})$, $\boldsymbol{\Omega}_{n}(\mathbf{k})=i\left\langle\nabla_{k} u_{n k}|\times| \nabla_{k} u_{n k}\right\rangle$ is the Berry curvature, $n$ is the band index, $\left|u_{n k}\right\rangle$ is the band wave function, and $\beta=1 /\left(k_{B} T\right)$.

In the considered case we have two bands (valence and conductivity) with $n= \pm, \quad \epsilon_{ \pm}(\mathbf{k})= \pm a, a=\sqrt{\hbar^{2} v_{F}^{2} \mathbf{k}^{2}+\Delta^{2}}$ and the wave function is

$$
\left|u_{ \pm k}\right\rangle=\frac{1}{\sqrt{2 a(a \mp \Delta)}}\left(\begin{array}{c}
\hbar v_{F}\left(k_{x}-i k_{y}\right) \\
\pm a-\Delta
\end{array}\right) .
$$

\subsection{Formal proof of the restoration of the third law of thermodynamics}

Employing the approach of Smrčka and Středa [51] developed for nonrelativistic fermions in a magnetic field, one can show that in general a part of the second term of Eq. (11) with $f(\epsilon)$ is cancelled by the magnetization (15). The term $\tilde{\beta}_{\alpha \beta}^{(2)}$ can be simplified using the relation

$$
\tau_{\alpha}=\frac{1}{i \hbar v_{F}}\left[r_{\alpha}, \mathcal{H}\right]=-\frac{1}{i \hbar v_{F}}\left[r_{\alpha}, G^{-1}\right]
$$

to the following form

$$
\begin{gathered}
\tilde{\beta}_{\alpha \beta}^{(2)}=-\frac{e v_{F}}{4 \pi i T} \int_{-\infty}^{\infty} d \epsilon f(\epsilon) \epsilon \times \\
\times \operatorname{Tr}\left[\frac{d\left(G^{R}-G^{A}\right)}{d \epsilon}\left(\hat{r}_{\beta} \tau_{\alpha}-\hat{r}_{\alpha} \tau_{\beta}\right)\right] .
\end{gathered}
$$

Integrating the last expression by parts we obtain

$$
\begin{gathered}
\tilde{\beta}_{\alpha \beta}^{(2)}=-\frac{e v_{F}}{4 \pi i T} \int_{-\infty}^{\infty} d \epsilon\left(-\frac{\partial f(\epsilon)}{\partial \epsilon}\right) \epsilon \times \\
\times \operatorname{Tr}\left[\left(G^{R}-G^{A}\right)\left(\hat{r}_{\beta} \tau_{\alpha}-\hat{r}_{\alpha} \tau_{\beta}\right)\right]+ \\
+\frac{e v_{F}}{4 \pi i T} \int_{-\infty}^{\infty} d \epsilon f(\epsilon) \operatorname{Tr}\left[\left(G^{R}-G^{A}\right)\left(\hat{r}_{\beta} \tau_{\alpha}-\hat{r}_{\alpha} \tau_{\beta}\right)\right] .
\end{gathered}
$$


Taking into account that $\delta(\epsilon-H)=-\left(G^{R}-G^{A}\right) / 2 \pi i$ one can see that the last term of Eq. (23) is equal to $-c M_{Z} / T$. Accordingly, it cancels out from the thermoelectric coefficient $\beta_{x y}$ defined by Eq. (8) and we arrive at the final expression

$$
\begin{gathered}
\beta_{\alpha \beta}=\frac{e \hbar v_{F}^{2}}{4 \pi T} \int_{-\infty}^{\infty} d \epsilon\left(-\frac{\partial f(\epsilon)}{\partial \epsilon}\right) \epsilon \times \\
\times \operatorname{Tr}\left\langle\tau_{\alpha} G^{R} \tau_{\beta}\left(G^{R}-G^{A}\right)-\tau_{\alpha}\left(G^{R}-G^{A}\right) \tau_{\beta} G^{A}\right\rangle_{c}- \\
-\frac{e v_{F}}{4 \pi i T} \int_{-\infty}^{\infty} d \epsilon\left(-\frac{\partial f(\epsilon)}{\partial \epsilon}\right) \epsilon \operatorname{Tr}\left\langle\left(G^{R}-G^{A}\right)\left(r_{\beta} \tau_{\alpha}-r_{\alpha} \tau_{\beta}\right)\right\rangle_{c} .
\end{gathered}
$$

Since Eq. (24) contains the derivative, $\partial f(\epsilon) / \partial \epsilon$, the thermoelectric coefficient vanishes in the limit $T \rightarrow 0$ as was already illustrated above. Writing down Eq. (24) we included the configurational averaging denoted as $\langle\cdots\rangle_{c}$. This takes care of the fact that the presented above proof is valid not only in the clean case, but for independent electrons in the presence of disorder. In a similar fashion one can show that the Hall conductivity is given by a similar expression without $\epsilon$ factor [57] (see also Ref. 58)

$$
\begin{gathered}
\sigma_{\alpha \beta}=\frac{e^{2} v_{F}^{2} \hbar}{4 \pi} \int_{-\infty}^{\infty} d \epsilon\left(-\frac{\partial f(\epsilon)}{\partial \epsilon}\right) \times \\
\times \operatorname{Tr}\left\langle\tau_{\alpha}\left(G^{R}-G^{A}\right) \tau_{\beta} G^{A}-\tau_{\alpha} G^{R} \tau_{\beta}\left(G^{R}-G^{A}\right)\right\rangle_{C}+ \\
+\frac{e^{2} v_{F}}{4 \pi i} \int_{-\infty}^{\infty} d \epsilon\left(-\frac{\partial f(\epsilon)}{\partial \epsilon}\right) \operatorname{Tr}\left\langle\left(G^{R}-G^{A}\right)\left(r_{\alpha} \tau_{\beta}-r_{\beta} \tau_{\alpha}\right)\right\rangle_{c} .
\end{gathered}
$$

This shows that the transport coefficients (including also the thermal conductivity) can be expressed via the electrical conductivity at $T=0$ [51]. This relationship between conductivity and other transport coefficients is valid under rather general assumptions, in particular, when the scattering of electrons is elastic.

Finally, after disorder averaging, the off-diagonal transport coefficients $\sigma_{x y}(\Delta)$ and $\beta_{x y}(\Delta)$ can be presented in the standard form

$$
\left\{\begin{array}{c}
\sigma_{x y} \\
\beta_{x y}
\end{array}\right\}=\frac{e^{2}}{\hbar} \int_{-\infty}^{\infty} d \epsilon\left[-\frac{\partial f(\epsilon)}{\partial \epsilon}\right]\left\{\begin{array}{c}
-1 \\
\frac{\epsilon}{e T}
\end{array}\right\} \mathcal{A}_{H}(\mu+\epsilon, \Delta)
$$

where all specific information about the model and the characteristics of elastic scattering is contained in the zero temperature Hall conductivity $\sigma_{x y}(\mu, \Delta, T=0)=$ $=-\left(e^{2} / \hbar\right) \mathcal{A}_{H}(\mu, \Delta)$ with the Hall kernel

$$
\begin{gathered}
\mathcal{A}_{H}(\mu+\epsilon, \Delta)= \\
=-\frac{\hbar^{2} v_{F}^{2}}{2 \pi} \int \frac{d^{2} k}{(2 \pi)^{2}} \operatorname{tr}\left[\tau_{1} G^{R}(\epsilon, \mathbf{k}) \mathbf{\Upsilon}_{2}^{R A}(\epsilon, \epsilon) G^{A}(\epsilon, \mathbf{k})\right],
\end{gathered}
$$

where the full vertex $\Upsilon_{\alpha}$ is for the particle current is defined as $\boldsymbol{\Upsilon}_{\alpha}^{(e)}=-e v_{F} \Upsilon_{\alpha}$.

\section{Results}

\subsection{Two-component Dirac fermions}

In the clean case, in the bubble approximation we take the bare vertex, $\Upsilon_{2}(\epsilon, \epsilon)=\tau_{2}$. Accordingly, the function $\mathcal{A}_{H}$ with the level broadening acquires the form

$$
\begin{gathered}
\mathcal{A}_{H}^{(c l)}(\epsilon, \Delta)=\frac{\Delta}{4 \pi^{2}}\left[\frac{1}{\epsilon}\left(\arctan \frac{|\Delta|+\epsilon}{\Gamma_{0}}-\arctan \frac{|\Delta|-\epsilon}{\Gamma_{0}}\right)+\right. \\
\left.+\frac{1}{|\Delta|}\left(\arctan \frac{\epsilon+|\Delta|}{\Gamma_{0}}-\arctan \frac{\epsilon-|\Delta|}{\Gamma_{0}}\right)\right]
\end{gathered}
$$

Accordingly, for $T \rightarrow 0$ (but $T \gg \Gamma_{0}$ ) we obtain

$$
\beta_{x y}^{(c l)}=-\beta_{0} \frac{\pi k_{B} T}{12} \frac{\Delta \operatorname{sgn}(\mu)}{\mu^{2}} \theta\left(\mu^{2}-\Delta^{2}\right),
$$

where $\beta_{0}=k_{B} e / \hbar$. It is easy to see that Eq. (29) also directly follows from the Mott relation (4) and the conductivity (5). However, the general expression (26) allows us to investigate the vicinity of the point $|\mu|=|\Delta|$, where the Mott result (29) fails.

The influence of disorder on the Hall conductivity of the gapped Dirac fermions was studied in [43,45]. We rederive in Appendix A this result by solving the equation for the dressed vertex $\Upsilon_{\alpha}^{(e)}$ in the ladder approximation. Substituting the vertex (A.17) in the Hall kernel $\mathcal{A}_{H}$ given by Eq. (28) we obtain

$$
\begin{aligned}
& \mathcal{A}_{H}^{(d)}(\epsilon, \Delta)=\frac{\operatorname{sgn}(\Delta)}{4 \pi} \theta\left(\Delta^{2}-\epsilon^{2}\right)+\frac{\Delta}{4 \pi|\epsilon|} \times \\
\times & {\left[1+\frac{4\left(\epsilon^{2}-\Delta^{2}\right)}{\epsilon^{2}+3 \Delta^{2}}+\frac{3\left(\epsilon^{2}-\Delta^{2}\right)^{2}}{\left(\epsilon^{2}+3 \Delta^{2}\right)^{2}}\right] \theta\left(\epsilon^{2}-\Delta^{2}\right) . }
\end{aligned}
$$

The important feature of $\mathcal{A}_{H}^{(d)}$ is that, in contrast to $\mathcal{A}_{H}^{(c l)}$, it is independent of the disorder potential strength and of the impurity concentration encoded in the scattering rates $\Gamma_{0}(\varepsilon)$ and $\Gamma_{1}(\varepsilon)$. Comparing the kernels $\mathcal{A}_{H}^{(d)}$ and $\mathcal{A}_{H}^{(c l)}$, one can see that the approximation of the disorder effects by the level broadening is insufficient even in the weak disorder limit $[43,45]$, and it leads to drastic changes in the behavior of $\sigma_{x y}$ and $\beta_{x y}$.

The dependences $\sigma_{x y}(\mu)$ and $\beta_{x y}(\mu)$ are plotted in the Fig. 2(a) and of Fig. 2(b), respectively. The dashed (red) and the solid (blue) curves correspond to the calculations 

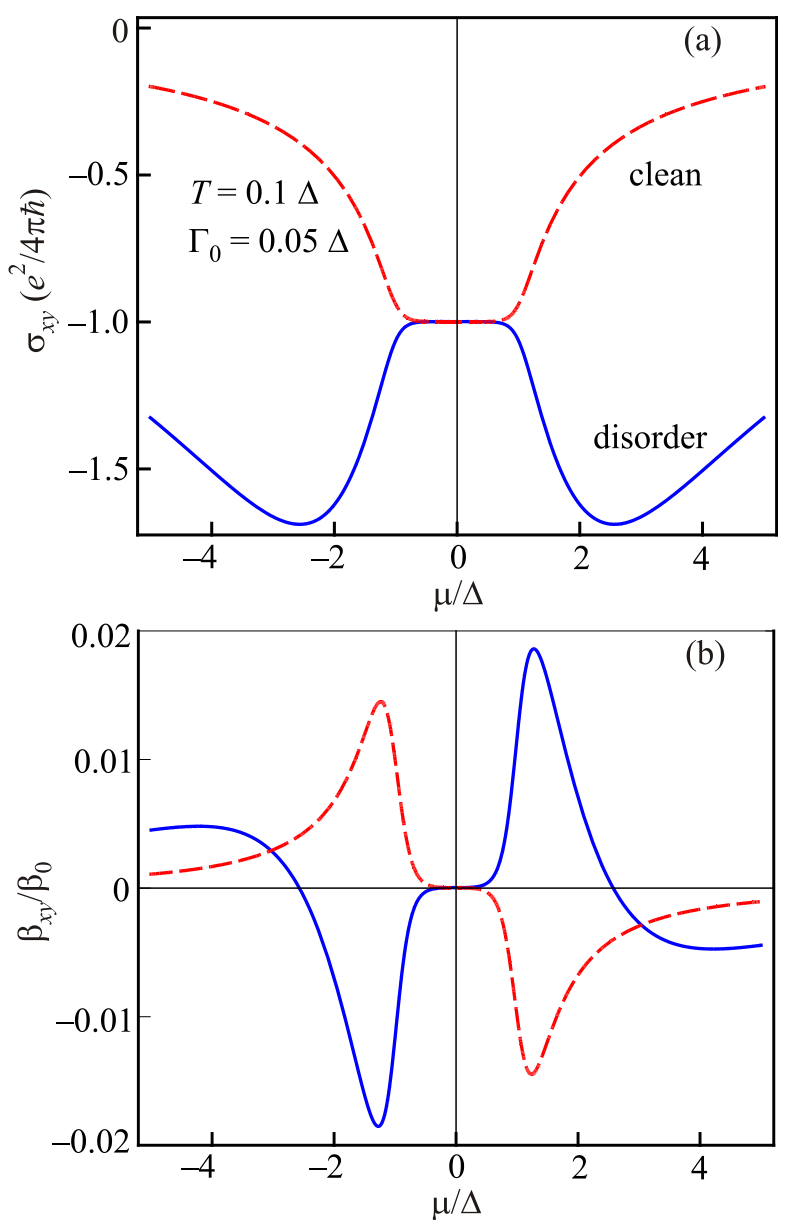

Fig. 2. (Color online) Electrical Hall conductivity $\sigma_{x y}(\mu)$ in units of $\sigma_{0}=e^{2} /(4 \pi \hbar)$ (a); thermoelectric coefficient $\beta_{x y}(\mu)$ in units of $\beta_{0}=k_{B} e / \hbar$ as functions of the chemical potential $\mu$ in the units of $\Delta>0$ (b).

done using the kernels $\mathcal{A}_{H}^{(d)}$ and $\mathcal{A}_{H}^{(c l)}$, respectively. We took $T=0.1 \Delta$ and $\Gamma_{0}=0.05 \Delta$. One can see that $\sigma_{x y}$ and $\beta_{x y}$ are even and odd functions of $\mu$, respectively. On the contrary, in the case of a real magnetic field, $\sigma_{x y}$ and $\beta_{x y}$ are odd and even functions of $\mu$, respectively. In this respect, a positive sign of the Nernst signal near $\mu=0$ is regarded as one of the fingerprints of the Dirac quasiparticles $[20,22]$. In the case of the anomalous Hall and Nernst effects, the sign of $\beta_{x y}$ also remains very informative. One can see that the presence of the disorder vertex drastically changes the pattern of the sign changes in $\beta_{x y}(\mu)$. The nonmonotonic dependence of $\sigma_{x y}(\mu)$ on the electron $(\mu>0)$ or hole $(\mu<0)$ parts of the carriers results in new nontrivial zeros of $\beta_{x y}(\mu)$. Using the Mott relation, one finds that these zeros are at $\mu= \pm \sqrt{3+2 \sqrt{3}}|\Delta| \approx \pm 2.5|\Delta|$.

\subsection{The results for silicene}

We return now to the discussion of the silicene model (1). We calculate the spin Hall conductivity $\sigma_{x y}^{S_{Z}}$ from Eq. (2) and the thermospin coefficient $\beta_{x y}^{S_{Z}}$ from its analog using Eq. (26) for the two-component Dirac fermions.

For reference, we begin with the kernel $\mathcal{A}_{H}^{(c l)}$. The spin Hall conductivity at $T=0$ and the zero sublattice asymmetry gap $\Delta_{Z}=0$ directly follows from Eq. (5) and reads [39,43]

$$
\begin{gathered}
\sigma_{x y}^{S_{Z}}=-\frac{e}{2 \pi} \operatorname{sgn}\left(\Delta_{S O}\right) \times \\
\times\left[\theta\left(\left|\Delta_{S O}\right|-|\mu|\right)+\frac{\left|\Delta_{S O}\right|}{|\mu|} \theta\left(|\mu|-\left|\Delta_{S O}\right|\right)\right] .
\end{gathered}
$$

Let us note that although the spin-orbit gap $\Delta_{\text {SO }}$ does not break the time-reversal symmetry, one can check that the Kubo contribution for $\Delta_{z}=0$ and $T \rightarrow 0$ is

$$
\tilde{\beta}_{x y}^{S_{Z}}=\frac{e}{\pi \hbar T} \Delta_{S O} \operatorname{sgn}(\mu), \quad|\mu|>\left|\Delta_{S O}\right| .
$$

This divergence, as above, is compensated by the "spin magnetization”

$$
M_{Z}^{S_{Z}}=-\frac{\hbar}{2 e} \sum_{\xi, \sigma= \pm} \xi \sigma M_{Z}\left(\Delta \rightarrow \Delta_{\xi \sigma}\right),
$$

which is nonzero even when the time-reversal symmetry is unbroken. Note that both the orbital magnetization

$$
M_{Z}=\sum_{\xi, \sigma= \pm} \xi M_{Z}\left(\Delta \rightarrow \Delta_{\xi \sigma}\right)
$$

and the electrical Hall conductivity

$$
\sigma_{x y}=\sum_{\xi, \sigma= \pm} \xi \sigma_{x y}\left(\Delta \rightarrow \Delta_{\xi \sigma}\right)
$$

in silicene in the absence of a magnetic field are equal to zero.

The final thermospin coefficient $\beta_{x y}^{S_{Z}}$ is given by Eq. (29) with $\Delta$ replaced by $\Delta_{S O}$ and $\beta_{0}$ by $\beta_{0}^{s}=k_{B} / 2$. Obviously, everything stated above concerning a large Nernst signal for the model (3) turns out to be applicable for the $\mathrm{SN}$ effect in silicene.

We present the dependences $\sigma_{x y}^{S_{Z}}(\mu)$ and $\beta_{x y}^{S_{Z}}(\mu)$ computed using the kernel (30) for a general case $\Delta_{Z} \neq 0$ in the Fig. 3(a),(b).

The case with the sublattice asymmetry gap $\Delta_{Z}=0.8 \Delta_{S O}$ is shown by the solid (blue) curves and the case $\Delta_{Z}=1.4 \Delta_{S O}$ is shown by the dashed (red) curves. We took the temperature $T=0.1 \Delta_{S O}$ that corresponds to $T \approx 10 \mathrm{~K}$ for silicene. We find that the presence of the disorder vertex resulted in a rather specific and rich pattern seen in the $\beta_{x y}^{S_{Z}}$ dependence, especially when the value of gap $\Delta_{Z}$ is closer to $\Delta_{S O}$. 

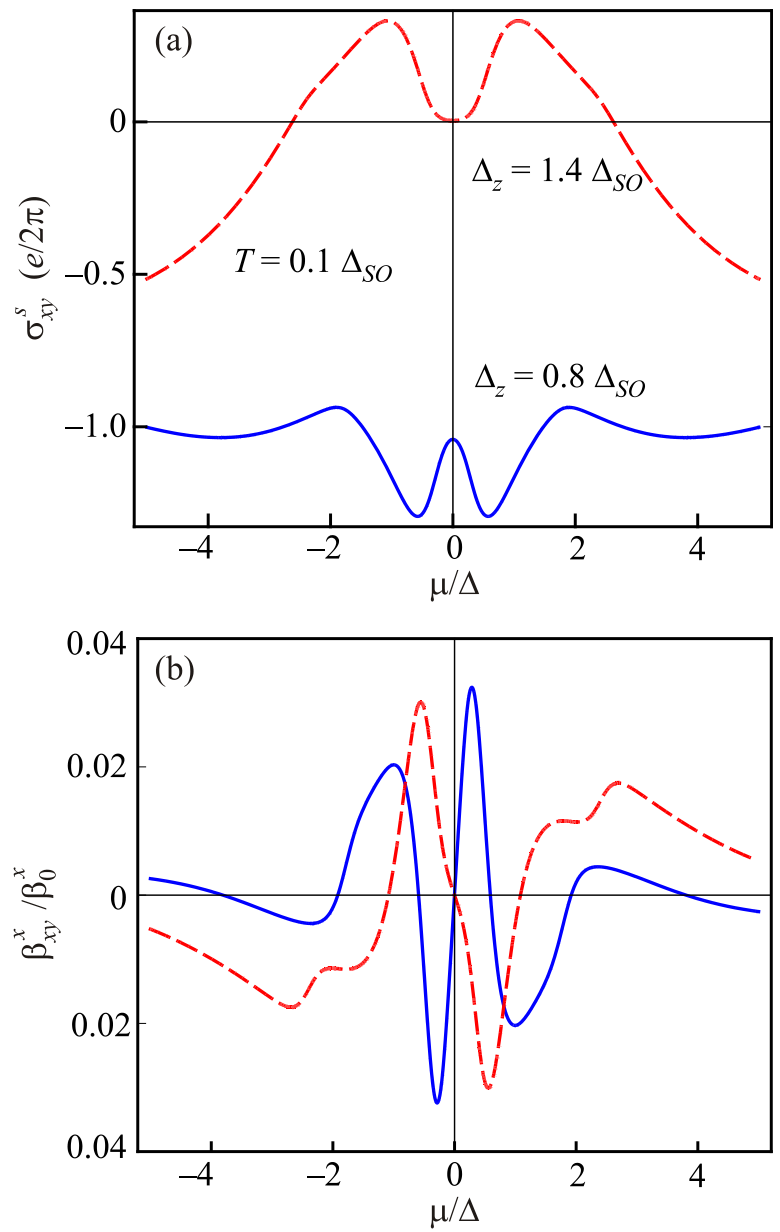

Fig. 3. (Color online) Spin Hall conductivity $\sigma_{x y} S_{Z}(\mu)$ in units of $\sigma_{0}^{S}=e /(2 \pi)$ (a); thermospin coefficient $\beta_{x y}^{S_{Z}}(\mu)$ in units of $\beta_{0}^{S}=k_{B} / 2$ as functions of the chemical potential $\mu$ in the units of $\Delta_{S O}>0$ (b).

It is instructive to represent the dependences of $\sigma_{x y}^{S_{Z}}$ and $\beta_{x y}^{S_{Z}}$ as the function of both of their variables $\mu$ and $\Delta_{Z}$ employing the density plot. Figures 4 and 5 are computed, respectively, using the kernel $\mathcal{A}_{H}^{(c l)}$ for the clean case and the kernel $\mathcal{A}_{H}^{(\mathrm{d})}$ for the case with disorder. One can see that in agreement with the analytical expressions for these kernels, the spin Hall conductivity $\sigma_{x y}^{S_{Z}}\left(\mu, \Delta_{z}\right)$ is even with respect to the variables $\mu$ and $\Delta_{z}$ both for the clean and disordered cases. On the other hand, the thermospin coefficient $\beta_{x y}^{S_{Z}}\left(\mu, \Delta_{z}\right)$ is odd with respect to $\mu$ and even with respect to $\Delta_{z}$ in both cases. Note that the spin Hall conductivity computed in the clean limit [Fig. 4(a)] is very similar to that found in Ref. 44 (Fig. 2).

It is worthwhile to stress the crucial role of disorder that dramatically changes the character of the dependences $\beta_{x y}^{S_{Z}}\left(\mu, \Delta_{z}\right)$ and $\sigma_{x y}^{S_{Z}}\left(\mu, \Delta_{z}\right)$ shown in Fig. 5. One can see that the number of the extrema near the points
( $\mu=0, \Delta_{z}= \pm \Delta_{S O}$ ) is duplicated with respect to the clean case. The same happens with the thermospin coefficient.

Similarly to the case of bilayer graphene, it should be possible in the experiments on silicene also to vary both $\mu$ and $\Delta_{z}$ independently and observe the predicted structures.

\section{Conclusions}

In the present work we studied the thermospin effect in silicene which is the base for other low-buckled Dirac materials such as germanene, stanene, and phosphorene. Neglecting the Rashba term in the Hamiltonian (1), we arrived at the simple but still nontrivial model of the twocomponent massive Dirac fermions. So far such an approach has allowed one to make a lot of progress in the analytical studies of the anomalous Hall effect [40]. Our study showed that anomalous thermoelectric transport can also be successfully investigated in this framework. We explicitly demonstrated how the standard Kubo formula has to be altered by including the effective magnetization leading to the correct off-diagonal thermoelectric coefficient. We also predicted a strong spin Nernst effect with nontrivial dependences on the carrier concentration and electric field applied in silicene and other low-buckled Dirac materials.

In conclusion, let us discuss the role of spin-nonconserving terms omitted in the model Hamiltonian (1). Actually, there are two different terms [42]. The first of the Rashba coupling contributions is associated with the nearest-neighbor hopping term and is induced by the external electric field $E_{z}$. The second term is associated with the next-nearest-neighbor hopping of electrons.

The first term in the continuum limit coincides with the Kane-Mele [39] Rashba term. In the clean limit, its impact on the SN effect is analyzed in detail in Ref. 46 using the Mott formula. In the existing literature on silicene, this term is neglected [42] because the corresponding coupling constant is two or three orders of magnitude less than the value of the second Rashba term.

With regard to the second Rashba coupling contribution, its effect is suppressed by the fact that it enters the dispersion law as the product of the coupling constant and momentum [42]. The impact of this Rashba term on the spin Hall conductivity was considered in Ref. 44. It is shown that in the reference case described by Eq. (31), the spin Hall conductivity is modified by the factor $v_{F}^{2} /\left(v_{F}^{2}+a^{2} \lambda_{R 2}^{2} / \hbar^{2}\right)$, where $\lambda_{R 2}$ is the coupling constant and $a$ is the lattice constant. Using that $v_{F}=\sqrt{3} /(2 \hbar) t a$ with $t \sim 1.6 \mathrm{eV}$ being the nearest neighbor hopping parameter, one can estimate that for the realistic values of $\lambda_{R 2} \sim 1 \mathrm{meV}$ the impact of the Rashba term on the spin Hall conductivity is indeed small, $\sim 10^{-6}$. Moreover, since this term does not affect the dependence of the spin Hall conductivity on $\mu$, the Mott formula would give the same result for the SN effect. Nevertheless, the role of the Rashba interactions, especially in the presence of the dressed by disorder vertex, should be thoroughly studied. 

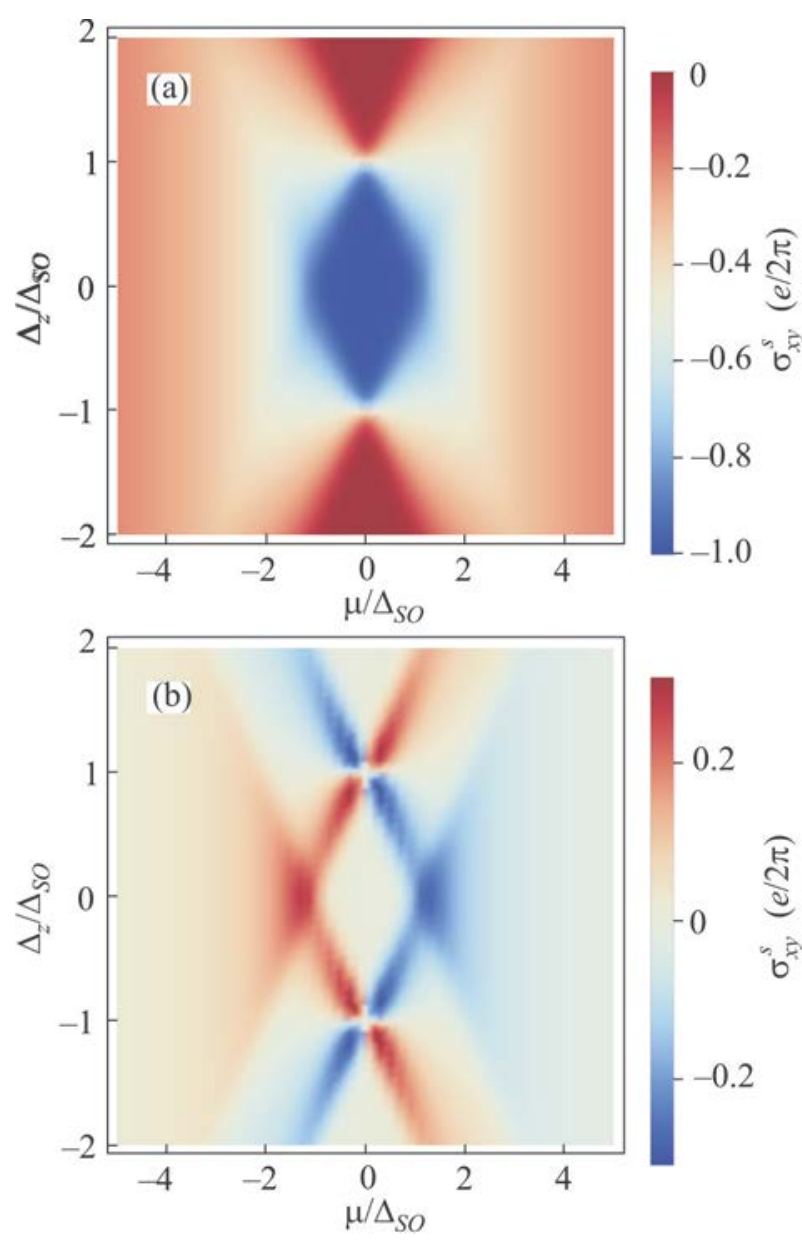

Fig. 4. (Color online) Spin Hall conductivity $\sigma_{x y}^{S_{Z}}$ in units of $\sigma_{0}^{S}$ (a); thermospin coefficient $\beta_{x y}^{S_{Z}}$ in units of $\beta_{0}^{S}$ as functions of the chemical potential $\mu$ and the sublattice asymmetry gap $\Delta_{Z}$ in the units of $\Delta_{S O}>0$ for the clean case (b).

The progress achieved in measuring spin currents (see e.g. Ref. 63 for a review) allows us to hope that the predicted very specific and strong SN effect in silicene can also be observed. At present theoretical studies of silicene and other related Dirac materials are ahead of the experimental ones. Silicene is only available on Ag and $\mathrm{ZrB}_{2}$ [30] substrate which are both conductive, there are no yet transport and optical measurements. Certainly the spintronics on silicene will only be possible when a more conventional transport experiment is performed.
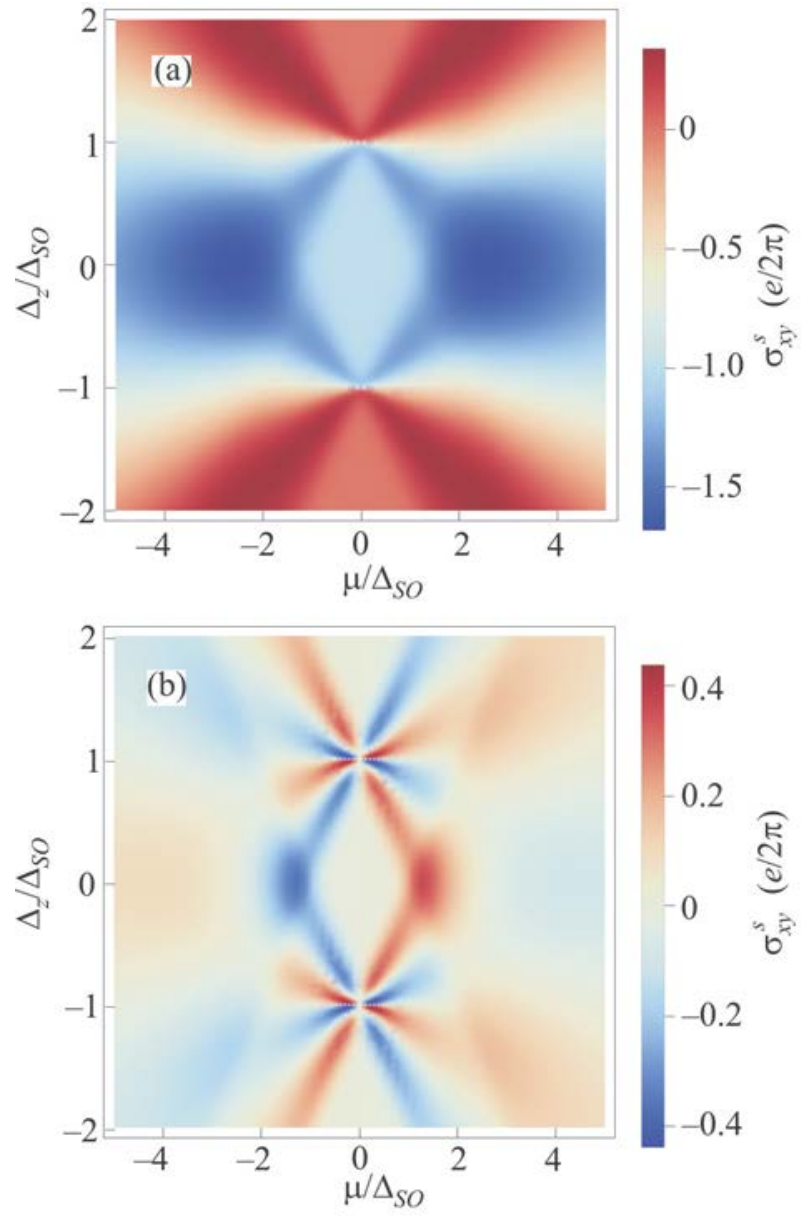

Fig. 5. (Color online) Spin Hall conductivity $\sigma_{x y}^{S_{Z}}$ in units of the value $\sigma_{0}^{S}$ (a); thermospin coefficient $\beta_{x y}^{S_{Z}}$ in units of the value $\beta_{0}^{S}$ as functions of the chemical potential $\mu$ and the sublattice asymmetry gap $\Delta_{Z}$ in the units of $\Delta_{S O}>0$ for the case with disorder (b).

\section{Acknowledgments}

The authors acknowledge the support of the European IRSES Grant SIMTECH No. 246937. The work V.P.G. and S.G.Sh. was supported partially by the Program of Fundamental Research of the Physics and Astronomy Division of the NAS of Ukraine.

\section{Appendix A: Electric current vertex for two-component Dirac fermions}

The dressed vertex $\Upsilon_{\alpha}^{(e)}\left(\epsilon_{n}+\Omega_{k}, \epsilon_{n} ; \mathbf{k}, \mathbf{k}\right)$ with zero external momentum $\mathbf{q}=0$ in the ladder approximation satisfies the equation

$$
\Upsilon_{\alpha}^{(e)}\left(\epsilon_{n}+\Omega_{m}, \epsilon_{n} ; \mathbf{k}, \mathbf{k}\right)=\Upsilon_{\alpha}^{(e)}+n_{\operatorname{imp}} \int \frac{d^{2} k^{\prime}}{(2 \pi)^{2}}\left|V\left(\mathbf{k}^{\prime}-\mathbf{k}\right)\right|^{2} G\left(\epsilon_{n}+\Omega_{m}, \mathbf{k}^{\prime}\right) \Upsilon_{\alpha}^{(e)}\left(\epsilon_{n}+\Omega_{m}, \epsilon_{n} ; \mathbf{k}^{\prime}, \mathbf{k}^{\prime}\right) G\left(\epsilon_{n}, \mathbf{k}^{\prime}\right)
$$

where $V(\mathbf{q})$ is the disorder potential, $n_{\text {imp }}$ is the concentration of impurities, and $\Upsilon_{\alpha}^{(e)}=-e v_{F} \tau_{\alpha}$ is the bare vertex. The full GF (10) includes the self-energy $\Sigma\left(\epsilon_{n}, \mathbf{p}\right)$ which is determined by the following self-consistent equation 


$$
\Sigma\left(\epsilon_{n}, \mathbf{k}\right)=n_{\text {imp }} \int \frac{d^{2} q}{(2 \pi)^{2}} V^{2}(\mathbf{q}) G\left(\epsilon_{n}, \mathbf{k}-\mathbf{q}\right) .
$$

We assume in what follows that the random disorder potential $V(\mathbf{r})$ is $\delta$-correlated (also spin-independent in the case of silicene) with Gaussian correlations

$$
\left\langle V\left(\mathbf{r}_{1}\right) V\left(\mathbf{r}_{2}\right)\right\rangle=n_{\mathrm{imp}} V^{2}(\mathbf{q}=\mathbf{0}) \delta\left(\mathbf{r}_{1}-\mathbf{r}_{2}\right),
$$

so that the self-energy and the vertex $\Upsilon_{\alpha}^{(e)}$ are independent of $\mathbf{k}$.

To calculate the transport coefficients we need the vertices $\Upsilon_{\alpha}^{(e) R A}$ and $\Upsilon_{\alpha}^{(e) R R}$ obtained by analytical continuation: $i \varepsilon_{n} \rightarrow \epsilon+i 0$ and $i \Omega_{m} \rightarrow \Omega \pm i 0$. Defining for convenience the vertex $\Upsilon_{\alpha}$ such that $\boldsymbol{\Upsilon}_{\alpha}^{(e)}=-e v_{F} \Upsilon_{\alpha}$, we obtain from Eq. (A.1) that the analytically continued vertex $\Upsilon_{\alpha}^{R X}$ with $X=R, A$ satisfies the equation

$$
\begin{gathered}
\Upsilon_{\alpha}^{R X}(\epsilon+\Omega, \epsilon)=\tau_{\alpha}+n_{\mathrm{imp}} V^{2}(\mathbf{0}) \times \\
\times \int \frac{d^{2} k^{\prime}}{(2 \pi)^{2}} G^{R}\left(\varepsilon+\Omega, \mathbf{k}^{\prime}\right) \Upsilon_{\alpha}^{R X}(\epsilon+\Omega, \epsilon) G^{X}\left(\epsilon, \mathbf{k}^{\prime}\right) .
\end{gathered}
$$

We seek for a solution of Eq. (A.3) in the following form

$$
\Upsilon_{\alpha}^{R X}(\epsilon+\Omega, \epsilon)=\sum_{\beta=0}^{3} Y_{\alpha \beta}^{R X} \tau_{\beta},
$$

where we omitted for brevity the arguments of the function $Y_{\alpha \beta}^{R X}$. Substituting the expansion (A.4) in Eq. (A.3), multiplying it by $\tau_{\gamma}$ and taking the $\operatorname{tr}$ we obtain the system of equations for the functions $Y_{\alpha \gamma}^{R X}$. The combinations

$$
\sum_{\beta=0}^{3} Y_{\alpha \beta}^{R X}(\epsilon+\Omega, \epsilon) \operatorname{tr}\left[\tau_{\gamma} G^{R}\left(\epsilon+\Omega, \mathbf{k}^{\prime}\right) \tau_{\beta} G^{X}\left(\epsilon, \mathbf{k}^{\prime}\right)\right]
$$

that enter the RHS of the equations can be simplified by expanding the GF over $\tau$-matrices:

$$
\begin{aligned}
& G^{R}(\varepsilon+\Omega, \mathbf{k})=g_{0}^{R} \tau_{0}+g_{1}^{R} k_{x} \tau_{1}+g_{2}^{R} k_{y} \tau_{2}+g_{3}^{R} \tau_{3}, \\
& G^{X}(\varepsilon, \mathbf{k})=g_{0}^{X} \tau_{0}+g_{1}^{X} k_{x} \tau_{1}+g_{2}^{X} k_{y} \tau_{2}+g_{3}^{X} \tau_{3} .
\end{aligned}
$$

Below we restrict ourselves by considering the $\Upsilon_{2}^{R X}$ component necessary for the calculation of the Hall coefficients. Noticing that the terms $\sim k_{x, y}$ anyway become zero after the momentum integration, one can safely omit them and obtain the traces

$$
\begin{gathered}
\frac{1}{2} \operatorname{tr}\left[\tau_{0} G^{R} \Upsilon_{2}^{R X} G^{X}\right]=\left(g_{0}^{R} g_{0}^{X}+g_{3}^{R} g_{3}^{X}+g_{1}^{R} g_{1}^{X} k_{x}^{2}+g_{2}^{R} g_{2}^{X} k_{y}^{2}\right) Y_{20}^{R X}+\left(g_{3}^{R} g_{0}^{X}+g_{0}^{R} g_{3}^{X}\right) Y_{23}^{R X}, \\
\frac{1}{2} \operatorname{tr}\left[\tau_{1} G^{R} \Upsilon_{2}^{R X} G^{X}\right]=\left(g_{0}^{R} g_{0}^{X}-g_{3}^{R} g_{3}^{X}+g_{1}^{R} g_{1}^{X} k_{x}^{2}-g_{2}^{R} g_{2}^{X} k_{y}^{2}\right) Y_{21} R X+i\left(g_{0}^{R} g_{3}^{X}-g_{3}^{R} g_{0}^{X}\right) Y_{22}^{R X}, \\
\frac{1}{2} \operatorname{tr}\left[\tau_{2} G^{R} \Upsilon_{2}^{R X} G^{X}\right]=-i\left(g_{0}^{R} g_{3}^{X}-g_{3}^{R} g_{0}^{X}\right) Y_{21}+\left(g_{0}^{R} g_{0}^{X}-g_{3}^{R} g_{3}^{X}-g_{1}^{R} g_{1}^{X} k_{x}^{2}+g_{2}^{R} g_{2}^{X} k_{y}^{2}\right) Y_{22} ; \\
\frac{1}{2} \operatorname{tr}\left[\tau_{3} G^{R} \Upsilon_{2}^{R X} G^{X}\right]=\left(g_{3}^{R} g_{0}^{X}+g_{0}^{R} g_{3}^{X}\right) Y_{20}+\left(g_{0}^{R} g_{0}^{X}+g_{3}^{R} g_{3}^{X}-g_{1}^{R} g_{1}^{X} k_{x}^{2}-g_{2}^{R} g_{2}^{X} k_{y}^{2}\right) Y_{23} .
\end{gathered}
$$

One can notice that the components $Y_{20}$ and $Y_{23}$ enter only Eqs. (A.6a) and (A.6d), so that the equation for vertex (A.3) has a solution with $Y_{20}=Y_{23}=0$. Then we obtain from Eq. (A.3) the following system of algebraic equations for the remaining components $Y_{21}, Y_{22}$ of the vertex

$$
\begin{aligned}
& {\left[A^{R X}(\epsilon+\Omega, \varepsilon)-1\right] Y_{21}^{R X}+C^{R X}(\epsilon+\Omega, \epsilon) Y_{22}^{R X}=0,} \\
& C^{R X}(\epsilon+\Omega, \epsilon) Y_{21}^{R X}+\left[1-A^{R X}(\epsilon+\Omega, \epsilon)\right] Y_{22}^{R X}=1,
\end{aligned}
$$

where the functions $A^{R X}$ and $C^{R X}$ are

$$
\begin{aligned}
& A^{R X}(\epsilon+\Omega, \epsilon)=n_{\mathrm{imp}} V^{2}(\mathbf{0}) \int \frac{d^{2} k}{(2 \pi)^{2}}\left[g_{0}^{R} g_{0}^{X}-g_{3}^{R} g_{3}^{X}\right], \\
& C^{R X}(\epsilon+\Omega, \epsilon)=n_{\mathrm{imp}} V^{2}(\mathbf{0}) \int \frac{d^{2} k}{(2 \pi)^{2}} i\left[g_{0}^{R} g_{3}^{X}-g_{3}^{R} g_{0}^{X}\right] .
\end{aligned}
$$

In obtaining the system (A.7) we used that

$$
\int \frac{d^{2} k}{(2 \pi)^{2}}\left[g^{R 1} g^{X 1} k_{x}^{2}-g^{R 2} g^{X 2} k_{y}^{2}\right]=0,
$$

because $g^{R 1} g^{X 1}=g^{R 2} g^{X 2}$ and depends on $k^{2}$ only. The formal solution of the system (A.7) reads

$$
\begin{aligned}
& Y_{21}^{R X}(\epsilon+\Omega, \epsilon)=\frac{C^{R X}(\epsilon+\Omega, \epsilon)}{\left(1-A^{R X}(\epsilon+\Omega, \epsilon)\right)^{2}+\left(C^{R X}(\epsilon+\Omega, \epsilon)\right)^{2}}, \\
& Y_{22}^{R X}(\epsilon+\Omega, \epsilon)=\frac{1-A^{R X}(\epsilon+\Omega, \epsilon)}{\left(1-A^{R X}(\epsilon+\Omega, \epsilon)\right)^{2}+\left(C^{R X}(\epsilon+\Omega, \epsilon)\right)^{2}} .
\end{aligned}
$$

One can easily see that in the clean limit $n_{\mathrm{imp}} V^{2}(\mathbf{0}) \rightarrow 0$ the functions $A^{R X}=C^{R X}=0$ and the bare vertex with $Y_{22}^{R X}=1$ is recovered. To obtain the vertex function in the generic case one should obtain the explicit expressions for the functions defined by Eq. (A.8). 
Calculation of the functions $A^{R X}$ and $C^{R X}$ and the final expression for the vertex function

To calculate the functions $A^{R X}$ and $C^{R X}$ we need the explicit expressions for the $g_{0,3}^{X}(\epsilon, \mathbf{k})$ components of the GF that enter the expansion (A.5). In its turn the full GF $G^{X}(\epsilon, \mathbf{k})$ depends on the self-energy $\Sigma^{X}(\epsilon)$ determined by the self-consistent equation (A.2) that can be be solved by iterations [23]. One can show that the terms $\sim \tau_{1,2}$ appear in the order $n_{\text {imp }}^{2}$ only, so that in the $\sim n_{\text {imp }}$ order

$$
\Sigma^{R}(\epsilon)=\tau_{0} \sigma_{0}^{R}(\epsilon)+\tau_{3} \sigma_{3}^{R}(\epsilon) .
$$

The real parts $\operatorname{Re} \sigma_{0}^{R}$ and $\operatorname{Re} \sigma_{3}^{R}$, which are logarithmically dependent on the high energy cutoff $W$, can be included in the renormalized $\mu$ and $\Delta$, respectively. We found that imaginary parts of the self-energy determining the quasiparticle relaxation rates $\Gamma_{0}=-\operatorname{Im} \sigma_{0}^{R}(\epsilon)$ and $\Gamma_{1}=-\operatorname{Im} \sigma_{3}^{R}(\epsilon)$, are

$$
\left\{\begin{array}{c}
\operatorname{Im} \sigma_{0}^{R}(\epsilon) \\
\operatorname{Im} \sigma_{3}^{R}(\epsilon)
\end{array}\right\}=-\frac{\theta\left[(\epsilon+\mu)^{2}-\Delta^{2}\right] \operatorname{sgn}(\epsilon+\mu)}{\left(\tau_{0} / \hbar\right)|\mu|}\left\{\begin{array}{c}
\epsilon+\mu \\
\Delta
\end{array}\right\} .
$$

Here we introduced the relaxation time scale

$$
\frac{1}{\tau_{0}}=\frac{n_{\mathrm{imp}} V(\mathbf{0})^{2}|\mu|}{4 \hbar^{3} v_{F}^{2}}
$$

Accordingly, the components of the GF entering the integrals (A.8) can be written in the form

$$
\begin{aligned}
& g_{0}^{R}(\epsilon, \xi)=\frac{\tilde{\epsilon}+i \Gamma_{0}}{\left(\tilde{\epsilon}+i \Gamma_{0}\right)^{2}-\xi^{2}-\left(\Delta-i \Gamma_{1}\right)^{2}}, \\
& g_{0}^{A}(\epsilon, \xi)=\frac{\tilde{\epsilon}-i \Gamma_{0}}{\left(\tilde{\epsilon}-i \Gamma_{0}\right)^{2}-\xi^{2}-\left(\Delta+i \Gamma_{1}\right)^{2}}, \\
& g_{3}^{R}(\epsilon, \xi)=\frac{\Delta-i \Gamma_{1}}{\left(\tilde{\epsilon}+i \Gamma_{0}\right)^{2}-\xi^{2}-\left(\Delta-i \Gamma_{1}\right)^{2}}, \\
& g_{3}^{A}(\epsilon, \xi)=\frac{\Delta+i \Gamma_{1}}{\left(\tilde{\epsilon}-i \Gamma_{0}\right)^{2}-\xi^{2}-\left(\Delta+i \Gamma_{1}\right)^{2}},
\end{aligned}
$$

where we denoted $\xi=\hbar v|\mathbf{k}|$ and $\tilde{\epsilon}=\epsilon+\mu$. Since we are interested in the dc transport, in what follows we restrict ourselves by the $\Omega=0$ case only. Both functions in (A.8) for the "RA" terms are expressed in terms of the same integral

$$
\begin{gathered}
I \equiv \frac{1}{2} \int_{0}^{\infty} \frac{d\left(\xi^{2}\right)}{\left(a-\xi^{2}\right)\left(a^{*}-\xi^{2}\right)}= \\
=\frac{1}{4\left|\tilde{\epsilon} \Gamma_{0}+\Delta \Gamma_{1}\right|}\left(\frac{\pi}{2}+\arctan \frac{\left.\tilde{\epsilon}^{2}-\Delta^{2}+\Gamma_{1}^{2}-\Gamma_{0}^{2}\right)}{2\left|\tilde{\epsilon} \Gamma_{0}+\Delta \Gamma_{1}\right|}\right) \approx \\
\approx \frac{\pi}{4\left|\tilde{\epsilon} \Gamma_{0}+\Delta \Gamma_{1}\right|}+O\left(\frac{\Gamma_{0,1}^{2}}{\tilde{\epsilon}^{2}}\right),
\end{gathered}
$$

where $a=\left(\tilde{\epsilon}+i \Gamma_{0}\right)^{2}-\left(\Delta-i \Gamma_{1}\right)^{2}$. Accordingly, the corresponding "RA" terms are

$$
\begin{aligned}
& A^{R A}(\epsilon, \epsilon)=\frac{n_{\mathrm{imp}} V(\mathbf{0})^{2}}{2 \pi \hbar^{2} v^{2}}\left(\tilde{\epsilon}^{2}+\Gamma_{0}^{2}-\Delta^{2}-\Gamma_{1}^{2}\right) I, \\
& C^{R A}(\epsilon, \epsilon)=-\frac{n_{\mathrm{imp}} V(\mathbf{0})^{2}}{\pi \hbar^{2} v^{2}}\left(\tilde{\epsilon} \Gamma_{1}+\Delta \Gamma_{0}\right) I .
\end{aligned}
$$

Following [45] it is convenient to introduce $\cos \theta=\Delta / \tilde{\epsilon}$ that allows to relate $\Gamma_{1}(\epsilon)$ and $\Gamma_{0}(\epsilon)$ via $\Gamma_{1}=\Gamma_{0} \cos \theta$. Then using Eqs. (A.11) and (A.12) we obtain

$$
\begin{gathered}
A^{R A}(\epsilon, \epsilon)=\frac{n_{\mathrm{imp}} V(\mathbf{0})^{2}|\tilde{\epsilon}|}{8\left(\hbar v_{F}\right)^{2} \Gamma_{0}} \frac{\sin ^{2} \theta}{1+\cos ^{2} \theta}=\frac{\sin ^{2} \theta}{2\left(1+\cos ^{2} \theta\right)}, \\
C^{R A}(\epsilon, \epsilon)=-\frac{n_{\mathrm{imp}} V(\mathbf{0})^{2}}{4\left(\hbar v_{F}^{2}\right)^{2}} \frac{\tilde{\epsilon} \Gamma_{1}+\Delta \Gamma_{0}}{\left|\tilde{\epsilon} \Gamma_{0}+\Delta \Gamma_{1}\right|}=-\frac{\Gamma_{0}}{\tilde{\epsilon}} \frac{2 \cos \theta}{1+\cos ^{2} \theta} .
\end{gathered}
$$

Here for brevity we omitted all $\theta$-functions that switch off $\Gamma_{0,1}$ when the energy $|\tilde{\epsilon}|<|\Delta|$. We note that since the integral (A.14) is proportional to $1 / n_{\mathrm{imp}} V(\mathbf{0})^{2}$, the small factor $n_{\mathrm{imp}} V(\mathbf{0})^{2}$ in (A.3) is cancelled out and the coefficient $A^{R A}$ turns out to be independent of the impurity strength and concentration. Then substituting the explicit expressions for $A^{R A}$ and $C^{R A}$ in the solution (A.10) and again expanding the result up to the same order $\sim \Gamma_{0,1} / \tilde{\epsilon}$ we arrive at the final expression for the vertex

$$
\Upsilon_{2}^{R A}(\epsilon, \epsilon)=-\tau_{x} \frac{8 \Gamma_{0} \Delta\left[(\epsilon+\mu)^{2}+\Delta^{2}\right]}{\left[(\epsilon+\mu)^{2}+3 \Delta^{2}\right]^{2}}+\tau_{y} \frac{2\left[(\epsilon+\mu)^{2}+\Delta^{2}\right]}{(\epsilon+\mu)^{2}+3 \Delta^{2}} .
$$

This agrees with the vertex found in Refs. 43, 45, where it was stressed that the vertex $\Upsilon_{2}^{R A}(\epsilon, \epsilon)=2 \tau_{y}$ is nontrivial even when $\Delta=0$.

A similar calculation for the "RR" term gives

$$
A^{R R}(\epsilon, \epsilon)=-\frac{\Gamma_{0}}{\pi|\epsilon+\mu|}, \quad C^{R R}(\epsilon, \epsilon)=0
$$

and, respectively, for the vertex one has

$$
\Upsilon_{2}^{R R}(\epsilon, \epsilon)=\tau_{y}\left(1-\frac{\Gamma_{0}}{\pi|\epsilon+\mu|}\right)
$$

1. V.P. Gusynin, S.G. Sharapov, and A.A. Varlamov, Phys. Rev. B 90, 155107 (2014).

2. K.L. Grosse, M.-H. Bae, F. Lian, E. Pop, and W.P. King, Nature Nanotechnology 6, 287 (2011).

3. T.T.M. Palstra, B. Batlogg, L.F. Schneemeyer, and J.V. Waszczak, Phys. Rev. Lett. 64, 3090 (1990).

4. M. Zeh, H.C. Ri, F. Kober, R.P. Huebener, A.V. Ustinov, J. Mannhart, R. Gross, and A. Gupta, Phys. Rev. Lett. 64, 3195 (1990). 
5. A.E. Koshelev, G. Logvenov, V. Larkin, V. Ryazanov, and K. Soifer, Physica C 177, 129 (1991).

6. Z.A. Xu, N.P. Ong, Y. Wang, T. Kakeshita, and S. Uchida, Nature, London 406, 486 (2000).

7. A Pourret, H. Aubin, J. Lesueur, C.A. Marrache-Kikuchi, and L. Berge, Nature Phys. 2 (10), 683 (2006).

8. M.A. Howson, M.B. Salamon, T.A. Friedmann, J.P. Rice, and D. Ginsberg, Phys. Rev. B 41, 300 (1990).

9. G.Yu. Logvenov, V.V. Ryazanov, R. Gross, and F. Kober, Phys. Rev. B 47, 15321 (1993).

10. H.C. Ri, R. Gross, F. Gollnik, A. Beck, R.P. Huebener, P. Wagner, and H. Adrian, Phys. Rev. B 50, 3312 (1994).

11. S. Ullah and A.T. Dorsey, Phys. Rev. B 44, 262 (1991).

12. I. Ussishkin, S.L. Sondhi, and D.A. Huse, Phys. Rev. Lett. 89, 287001 (2002).

13. Yu.N. Obtaztsov, Sov. Phys. Solid State 6, 331 (1964).

14. E.H. Sondheimer, Proc. R. Soc. A 193, 484 (1948).

15. G. Mahan, Many-Particle Physics, Springer (2000).

16. M. Serbyn, M.A. Skvortsov, A.A. Varlamov, and V.M. Galitsky, Phys. Rev. Lett. 102, 067001 (2009).

17. N.R. Cooper, B.I. Halperin, and I.M. Ruzin, Phys. Rev. B 55, 2344 (1997).

18. P. Wei, W. Bao, Y. Pu, C.N. Lau, and J. Shi, Phys. Rev. Lett. 102, 166808 (2009).

19. Y.M. Zuev, W. Chang, and P. Kim, Phys. Rev. Lett. 102, 096807 (2009).

20. J.G. Checkelsky and N.P. Ong, Phys. Rev. B 80, 081413 (2009).

21. D. Wang and J. Shi, Phys. Rev. B 83, 113403 (2011).

22. V.P. Gusynin and S.G. Sharapov, Phys. Rev. B 73, 245411 (2006).

23. S.G. Sharapov and A.A. Varlamov, Phys. Rev. B 86, 035430 (2012).

24. C.-R. Wang, W.-S. Lu, L. Hao, W.-L. Lee, T.-K. Lee, F. Lin, I.-C. Cheng, and J.-Z. Chen, Phys. Rev. Lett. 107, 186602 (2011).

25. B. Lalmi, H. Oughaddou, H. Enriquez, A. Kara, S. Vizzini, B. Ealet, and B. Aufray, Appl. Phys. Lett. 97, 223109 (2010).

26. P. De Padova, C. Quaresima, C. Ottaviani, P.M. Sheverdyaeva, P. Moras, C. Carbone, D. Topwal, B. Olivieri, A. Kara, H. Oughaddou, B. Aufray, and G. Le Lay, Appl. Phys. Lett. 96, 261905 (2010).

27. P. De Padova, C. Quaresima, B. Olivieri, P. Perfetti, and G. Le Lay, Appl. Phys. Lett. 98, 081909 (2011).

28. P. Vogt, P. De Padova, C. Quaresima, J. Avila, E. Frantzeskakis, M.C. Asensio, A. Resta, B. Ealet, and G. Le Lay, Phys. Rev. Lett. 108, 155501 (2012).

29. C.-L. Lin, R. Arafune, K. Kawahara, N. Tsukahara, E. Minamitani, Y. Kim, N. Takagi, and M. Kawai, Appl. Phys. Express 5, 045802 (2012).

30. A. Fleurence, R. Friedlein, T. Ozaki, H. Kawai, Y. Wang, and Y. Yamada-Takamura, Phys. Rev. Lett. 108, 245501 (2012).

31. L. Chen, C.-C. Liu, B. Feng, X. He, P. Cheng, Z. Ding, S. Meng, Y. Yao, and K. Wu, Phys. Rev. Lett. 109, 056804 (2012).

32. Z. Majzik, M.R. Tchalala, M. Svec, P. Hapala, H. Enriquez, A. Kara, A.J. Mayne, G. Dujardin, P. Jelínek, and H. Oughaddou, J. Phys.: Cond. Mat. 25, 225301 (2013).
33. S. Cahangirov, M. Topsakal, E. Aktürk, H. Şahin, and S. Ciraci, Phys. Rev. Lett. 102, 236804 (2009).

34. N.D. Drummond, V. Zólyomi, and V.I. Falko, Phys. Rev. B 85, 075423 (2012).

35. C.-C. Liu, W. Feng, and Y. Yao, Phys. Rev. Lett. 107, 076802 (2011).

36. C.-C. Liu, H. Jiang, and Y. Yao, Phys. Rev. B 84, 195430 (2011).

37. W.-F. Tsai, C.-Y. Huang, T.-R. Chang, H. Lin, H.-T. Jeng, and A. Bansil, Nature Commun. 4, 1500 (2013).

38. Y. Xu, B. Yan, H.-J. Zhang, J. Wang, G. Xu, P. Tang, W. Duan, and S.-C. Zhang, Phys. Rev. Lett. 111, 136804 (2013).

39. C.L. Kane and E.J. Mele, Phys. Rev. Lett. 95, 146802 (2005); ibid. 95, 226801 (2005).

40. N. Nagaosa, J. Sinova, S. Onoda, A.H. MacDonald, and N.P. Ong, Rev. Mod. Phys. 82, 1539 (2010).

41. G.E.W. Bauer, E. Saitoh, and B.J. van Wees, Nat. Mat. 11, 391 (2012).

42. M. Ezawa, New J. Phys. 14, 033003 (2012); Phys. Rev. Lett. 109, 055502 (2012).

43. N.A. Sinitsyn, J.E. Hill, H. Min, J. Sinova, and A.H. MacDonald, Phys. Rev. Lett. 97, 106804 (2006).

44. A. Dyrdał and J. Barnaś, Phys. Status Solidi (RRL) 6, 340 (2012).

45. N.A. Sinitsyn, A.H. MacDonald, T. Jungwirth, V.K. Dugaev, and J. Sinova, Phys. Rev. B 75, 045315 (2007).

46. Z. Guo-Bao, Chin. Phys. B 21, 117309 (2012).

47. A. Dyrdał and J. Barnaś, J. Phys. Cond. Matt. 24, 275302 (2012).

48. D.G. Rothe, E.M. Hankiewicz, B. Trauzettel, and M. Guigou, Phys. Rev. B 86, 165434 (2012).

49. X. Liu and X.C. Xie, Solid State Commun. 150, 471 (2010).

50. Yu.N. Obraztsov, Fiz. Tverd. Tela 6, 414 (1964) [Sov. Phys. Solid State 6, 331 (1964)]; ibid. 7, 573 (1965).

51. L. Smrčka and P. Středa, J. Phys. C 10, 2153 (1977); P. Středa, J. Phys. C 15, L717 (1982).

52. M. Jonson and S.M. Girvin, Phys. Rev. B 29, 1939 (1984).

53. H. Oji and P. Streda, Phys. Rev. B 31, 7291 (1985).

54. N.R. Cooper, B.I. Halperin, and I.M. Ruzin, Phys. Rev. B 55, 2344 (1997).

55. Di Xiao, Y. Yao, Z. Fang, and Q. Niu, Phys. Rev. Lett. 97, 026603 (2006).

56. A. Larkin and A. Varlamov, Theory of Fluctuations in Superconductors, Oxford University Press (2005).

57. A. Bastin, C. Lewiner, O. Betbeder-Matibet, and P. Noziers, J. Phys. Chem. Solids 32, 1811 (1971).

58. A. Crépieux and P. Bruno, Phys. Rev. B 64, 014416 (2001).

59. K.-T. Chen and P.A. Lee, Phys. Rev. B 84, 205137 (2011).

60. J. Shi, G. Vignale, Di Xiao, and Q. Niu, Phys. Rev. Lett. 99, 197202 (2007).

61. D. Xiao, M.-C. Chang, and Q. Niu, Rev. Mod. Phys. 82, 1959 (2010).

62. R. Nourafkan, G. Kotliar, and A.-M.S. Tremblay, Phys. Rev. B 90, 125132 (2014).

63. S. Maekawa, H. Adachi, K. Uchida, J. Ieda, and E. Saitoh, J. Phys. Soc. Jpn. 82, 102002 (2013). 